\title{
Engagement fällt nicht vom Himmel
}

\author{
Thomas Mäule
}

Das Profil von freiwilligen Mitarbeiterinnen und Mitarbeitern in Pflegeheimen weicht markant vom Bürgerengagement der Gesamtbevölkerung ab: Doch lassen sich auch zwischen den in der stationären Altenpflege freiwillig Engagierten große Unterschiede feststellen, wie eine Umfrage der Evangelischen Heimstiftung in Baden-Württemberg ergeben hat. Die sehr unterschiedlichen Motive liefern für das Freiwilligenmanagement wichtige Hinweise: Passgenaue Angebote sind gefragt, die ausdrücklich Interessen und Motive von Ehrenamtlichen ansprechen.

Die Mitarbeit von freiwillig Engagierten ist ein wesentlicher Bestandteil Sozialer Arbeit und verleiht ihr eine besondere Qualität: Die Lebensqualität von Bewohnern, Patienten und Klienten wird erhöht. Leistungen sind möglich, die aus Zeitmangel oder Kostengründen nicht angeboten werden können.

Freiwillige sind unabhängiger als die beruflichen Fachkräfte. Sie sind das »Gewissen « von Einrichtungen. Sie erkennen Probleme oft lange bevor sie von Hauptamtlichen wahrgenommen werden, sie bringen neue Ideen ein und helfen, neue Betreuungsleistungen zu konzipieren. Ehrenamtliche sind »Anwälte« pflegebedürftiger Menschen und »Fürsprecher« für eine menschenwürdige Hilfe. Sie sind »freiwillige Experten «, bringen spezifische Kenntnisse und professionelle Fähigkeiten mit. Sie stehen in Kontakt mit der Umwelt der Organisation, erhöhen die Kundenbindung, begünstigen Einnahmen in Form von Spenden und Fördergeldern. Modellprojekte zeigen, dass der Einsatz von Freiwilligen nicht nur ein strategischer Vorteil für Einrichtungen darstellt, sondern die Wertschöpfung durch Freiwilligenarbeit die Kosten eines systematischen Freiwilligenmanagements auch deutlich übersteigt.

Die Etablierung einer professionellen Freiwilligenkoordination hat allerdings in Deutschland kaum stattgefunden. Im Fünften Altenbericht der Bundesregierung wird dargelegt, »dass die Einrichtungen i. d. R. keine systematischen Konzepte für die Einbeziehung der Freiwilligen haben, so dass hier noch ein großer Entwicklungsbedarf besteht « (BMFSFJ 2005, 351). Demgemäß sind Einrichtungen gefordert, den strukturellen und organisatorischen Rahmen für ein Freiwilligenmanagement zu konzeptionalisieren.

Aktuelle Untersuchungen belegen, dass die Bereitschaft, sich freiwillig und unbezahlt für andere zu engagieren,

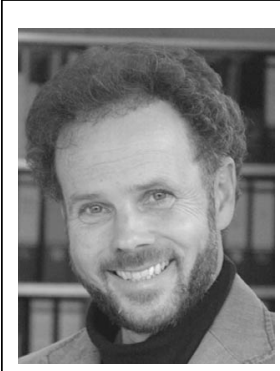

Dr. Thomas Mäule studierte Theologie, Sportwissenschaft, Gerontologie und Sozialmanagement. Er war Gemeindepfarrer, Studienleiter an der Evangelischen Akademie Bad Boll und Leiter des Grundsatzreferates im Diakonischen Werk Württemberg. Derzeit ist er Referent für Theologie und Ethik bei der Evangelischen Heimstiftung GmbH in Stuttgart. Die Organisation ist mit 6.800 Plätzen der größte Anbieter von Altenhilfedienstleistungen in Baden-Württemberg. In 61 Alten- und Pflegeheimen arbeiten rund 5.500 Beschäftigte.

E-Mail t.maeule@ev-heimstiftung.de

nach wie vor vorhanden ist, und die produktiven Potenziale in allen Altersgruppen bei weitem noch nicht ausgeschöpft sind (vgl. BMFSFJ 2005, 339; H. Hoch u. a. 2006, 56). Umfangreiche Studien enthalten wichtige Daten zu Engagement und Ehrenamt, Trends und Veränderungen. (1) Dies darf aber nicht darüber hinwegtäuschen, dass unklar ist, ob die darin beschriebenen Orientierungen und Entwicklungen grundsätzlich für alle Engagementbereiche und auch für die Arbeit in kirchlichen Wohlfahrtsverbänden gelten. Fraglich ist, ob aus jenen Erhebungen empirisch sichere Rückschlüsse auf freiwilliges soziales Engagement in der stationären Pflege und in einem milieuorientierten Kontext möglich sind. Bislang liegen keine repräsentativen Untersuchungen in diakonischen Pflegeeinrichtungen über Art und Umfang, Motive und Einstellungen, Probleme und Perspektiven von Ehrenamtlichen vor.

Vor diesem Hintergrund hat die Geschäftsführung der Evangelischen Heimstiftung Stuttgart GmbH beschlossen, eine Repräsentativbefragung unter allen 2.019 Ehrenamtlichen durchzuführen, die von 58 Pflegeeinrichtungen gemeldet wurden. Neben demografischen und grundsätzlichen Strukturdaten sollten Motive und Einstellungen von Freiwilligen in Erfahrung gebracht werden, um verlässliche Daten für die künftige Ausrichtung der Ehrenamtsarbeit und Impulse für die Gestaltung eines Freiwilligenmanagements zu haben. Die Befragungsergebnisse werden im Folgenden dargestellt sowie inhaltliche Anknüpfungspunkte und Gestaltungsempfehlungen für das Freiwilligenmanagement aufgezeigt. 


\section{Die Ergebnisse}

Die Befragung der Evangelischen Heimstiftung wurde als Vollerhebung konzipiert, der Fragebogen von Mitarbeitenden der Organisation unter Einschluss von Ehrenamtlichen gemeinsam mit der Gesellschaft für Marketingforschung »Konzept \& Markt« konzipiert. Dabei sollten folgende Untersuchungsziele verfolgt werden:

- Kenntnis über Art und Vielfalt des Engagements

- Kenntnis über die Motive des Engagements

- Schwierigkeiten, die den Ehrenamtlichen begegnen

- Evaluation von Impulsen für bessere Unterstützung

- Hinweise, wie weitere Ehrenamtliche zur Mitarbeit gewonnen werden können

Die Befragung fand vom 5. Mai bis 15. Juni 2008 statt. In den Einrichtungen übergaben die Hausdirektionen die Befragungsunterlagen persönlich an die Ehrenamtlichen. Die Unterlagen erhielten einen verschließbaren, fensterlosen Rückumschlag, der portofrei an die Gesellschaft für Marketingforschung »Konzept \& Markt« in Wiesbaden adressiert war.

Der Fragebogen enthielt offene, halboffene und geschlossene Fragen. Die Auswertung erfolgte grundsätzlich quantitativ und in Form einfacher, beschreibender Statistik. Eine Ausnahme bildete der Fragenkomplex zu der Motivation. Hier wurde eine Clusteranalyse angewandt, um »Motivationstypen « abzuleiten. Die Hypothese ist, dass von einer begrenzten Anzahl nebeneinander existierender Formen und Typologien ehrenamtlichen Engagements auszugehen ist. Diese Typen sollten herausgefunden, näher beschrieben und quantifiziert werden.

Zum Abschluss der Erhebung lagen 1.020 Fragebögen vor. Die Rücklaufquote von 50,7 Prozent entspricht - gegenüber vergleichbaren Studien - einem außerordentlich hohen Respons. Mit der schriftlichen Befragung liegen repräsentative Informationen über Ehrenamtliche in der stationären Pflege vor.

\section{Merkmale des Engagements}

Lebensalter, zeitliche Beanspruchung in Beruf oder Familie und ehrenamtliches Engagement hängen bei der Evangelischen Heimstiftung eng zusammen. Für viele ist ein Ehrenamt nur denkbar, wenn andere Anforderungen und Verpflichtungen weniger wichtig werden. Insbesondere sind es ältere Ehrenamtliche, die sich bei der Evangelischen Heimstiftung engagieren: Bei der Altersverteilung sind 34 Prozent zwischen 60 und 69 Jahren, 35 Prozent sind 70 Jahre und älter. Der Altersgruppe der 50- bis 59jährigen gehören 13 Prozent, der 40- bis 49jährigen fünf Prozent an. Nur zwei Prozent sind jünger als 40 Jahre. 75 Prozent der freiwillig Engagierten sind Frauen.

Dass das Engagement eng mit Lebensphasen zusammenhängt, bestätigen die Strukturdaten zur Erwerbstätig- keit. Die größte Gruppe der freiwillig Engagierten sind Rentner. Der Altersstruktur entsprechend sind 74 Prozent der freiwillig Engagierten nicht (mehr) erwerbstätig. Der Anteil der Berufstätigen liegt bei den Männern bei 16 Prozent, bei den Frauen bei 17 Prozent. Was den Familienstand betrifft, sind 62 Prozent der Ehrenamtlichen verheiratet, 20 Prozent sind verwitwet, elf Prozent sind ledig und sieben Prozent geschieden.

Das Engagement in der stationären Pflege ist weitgehend unabhängig davon, ob Angehörige in der Pflegeeinrichtung leben. Bei sechs Prozent der Engagierten wohnt aktuell eine Bezugsperson in der Einrichtung, 18 Prozent hatten früher pflegebedürftige Familienangehörige im Heim. Der größte Teil der Ehrenamtlichen (71\%) engagiert sich dagegen ohne Verwandtschaftsbezug.

Wichtig für freiwilliges Engagement ist die sozialräumliche Nähe der Ehrenamtlichen zur Pflegeeinrichtung. 35 Prozent der Ehrenamtlichen erreichen ihren Einsatzbereich innerhalb von 15 Gehminuten. 39 Prozent haben eine Wegstrecke von bis zu fünf Kilometern. Das freiwillige Engagement speist sich aus dem Nahraum. Weite Strecken minimieren die Engagementbereitschaft.

Vergleicht man die soziodemografischen Merkmale mit vergleichbaren Studien, so weicht das Profil der Freiwilligen in Pflegeheimen markant vom Bürgerengagement der Gesamtbevölkerung ab: Auffallend in der stationären Pflege ist die Dominanz der Frauen, während Männer deutlich unterrepräsentiert sind. Im Blick auf die Altersgruppen sind die unter 40- und unter 50jährigen kaum präsent. Dagegen ist die Gruppe der über 60 und über 70jährigen, der Verheirateten, Verwitweten und nicht mehr Erwerbstätigen überdurchschnittlich vertreten.

Auch hinsichtlich der konfessionellen Orientierung unterscheiden sich die Ehrenamtlichen der Evangelischen Heimstiftung zum Teil deutlich von den in anderen Milieus Engagierten. Ehrenamtliche der Evangelischen Heimstiftung sind durch eine enge Verbundenheit zu evangelischer oder katholischer Kirche geprägt. 90 Prozent der Ehrenamtlichen gehören einer der beiden großen Kirchen an (63\% evangelisch, 27\% katholisch). Die kirchliche Orientierung spielt für das Engagement eine bedeutende Rolle. Die große Mehrheit beschreibt sich als kirchenverbunden: Für fast zwei Drittel bedeutet die Zugehörigkeit der Pflegeeinrichtung zur Kirche viel (27\% sehr wichtig, 36\% wichtig). Nur eine Minderheit geht auf Distanz. So betonen nur elf Prozent, dass ihnen die konfessionelle Bindung nichts bedeute.

Allerdings zeigt die Analyse auch, dass die Kirchenbindung tendenziell als Einflussfaktor an Bedeutung verliert. Bei jüngeren Ehrenamtlichen ist der konfessionelle Hintergrund deutlich geringer ausgeprägt. Zwar bekräftigt eine knappe Mehrheit der unter 50jährigen ihre generelle Kirchenverbundenheit; aber jeder vierte der unter 40jährigen formuliert seine Position: »Die Zugehörigkeit meiner Einrichtung zur Kirche ist für mein Engagement unwichtig.« Ganz offensichtlich besteht ein Zusammenhang zwischen Alter und kirchlicher Orientierung. Mit zunehmendem Alter steigt (linear) die Bedeutung der konfessionellen Bindung. 
Im Mittelpunkt des freiwilligen Engagements steht die persönliche Begegnung mit Menschen, die in stationären Einrichtungen leben. Um diesen Kern des Engagements bildet sich ein weites Tätigkeitsspektrum, das kulturelle und religiöse Angebote, Sterbebegleitung, Hol- und Bringdienste etc. umfasst (vgl. Abb. 1).
In hohem Ausmaß ist das Gefühl verbreitet, nicht nur selbst Zeit und Kraft zu investieren, sondern umgekehrt selbst vom Ehrenamt zu profitieren. So geht für 41 Prozent die Tätigkeit mit der Chance einher, Kontakte zu anderen Menschen zu knüpfen. Für 54 Prozent ist ihr Engagement zudem eine Tätigkeit, die ihrem Leben Sinn gibt. Auch die Absicht, sich mit seinem Engagement weiterzuentwickeln, neue Erfahrungen zu machen und neue Kompetenzen zu erwerben, wird als wichtiger Beweggrund genannt (27\%). Vor allem bei jüngeren Altersgruppen hat dieses Motiv eine hervorgehobene Bedeutung (bei den bis 40Jährigen: $52 \%$, bei den über 70Jährigen dagegen nur 19\%). 15 Prozent bekennen, dass ihr Engagement auch zur Selbstverwirklichung beiträgt und die Chance bietet, Angelegenheiten selbst in die Hand zu nehmen, zu organisieren und umzusetzen. Die jüngste Altersgruppe nennt diese Absicht viermal so oft wie die älteste.
Die Zugangswege zum Ehrenamt zeigen, dass insbesondere die persönliche Ansprache - von haupt- und ehrenamtlichen Mitarbeitern - den Anstoß zum freiwilligen Engagement gegeben hat. Ehemalige Mitarbeitende (in der Altersgruppe zwischen 60 und 70 Jahren) melden sich auffallend häufig.

Bei der Frage nach den Beweggründen, die zum Engagement geführt haben, steht an erster Stelle: die Lebensqualität der Bewohner zu verbessern (73\%). Mehr als der Hälfte der Freiwilligen macht ihre Arbeit Spaß und Freude (55\%). Die jüngeren Altersgruppen messen dem Motiv Spaß und Freude ein deutlich höheres Gewicht zu als die älteren.

Neben altruistischen Motiven wie dem Wunsch, pflegebedürftigen Menschen zu helfen und selbstverständlicher Pflichtwertorientierung spielen aber auch Gründe eine Rolle, die auf eigene Interessen und Bedürfnisse abzielen.

\section{Eine Typologie}

Den typischen Ehrenamtlichen, der einem einheitlichen Bild vom Ehrenamt entspricht, scheint es in der Evangelischen Heimstiftung nicht zu geben. Dies entspricht der Diskussionslage der Forschung, die von einer Vielzahl gleichzeitig nebeneinander existierender Formen und Typologien ehrenamtlichen Engagements ausgeht. Die These von der Verlagerung von Motivstrukturen weg von Pflicht- und hin zu Selbstentfaltungswerten ist ebenso wenig zutreffend wie die Annahme eines Übergangs vom »alten« zum »neuen« Ehrenamt.

Selten gibt es ein einziges handlungsleitendes Motiv, etwa »Spaß haben«, meist wirken mehrere Motive zusammen: »Ich habe Spaß, alten Menschen zu helfen. «Sinnvoll ist es deshalb, von Motivbündeln zu sprechen.

Bündelt man die Antworten der Befragung in der Evangelischen Heimstiftung durch eine Faktorenanalyse, so zeigt sich, dass solche Motivbündel in allen Kombinationen bei den Engagierten vorkommen. Darüber hinaus gibt es ein zentrales Motiv, das weitgehend von allen Ehrenamtlichen genannt wird: »die Lebensqualität der Bewohner zu verbessern $«$. Diese Hauptmotivation ist generell und tritt in allen Motivationsprofilen auf. $\mathrm{Zu}$ diesem Kern kommen dann weitere - ergänzende Motivbündel hinzu. Sechs unterscheidbare Ehrenamtsgruppen lassen sich durch die Clusteranalyse ausmachen (vgl. Abb. 2). 
Die größte Gruppe der Ehrenamtlichen in der Evangelischen Heimstiftung (mit einem Anteil von 43\%) ist von ihren Einstellungen her stark an traditions- und normgeleiteten Motiven orientiert. Sie ist aktiv in die Kirchengemeinde eingebunden und versteht ihr Engagement als Teil kirchlichen Handelns. In Anlehnung an die wissenschaftliche Diskussion werden diese ehrenamtlich Engagierten, die das Rückgrat der Freiwilligen in der Organisation bilden, hier als »milieuverbunden Pflichtorientierte bezeichnet (vgl. Abb. 3).

\begin{tabular}{|c|c|}
\hline Engagementgruppe & Milieuverbunden Pflichtorientierte \\
\hline Anteil & $43 \%$ \\
\hline $\begin{array}{l}\text { Motivbündel/Gründe } \\
\text { für ehrenamtliches } \\
\text { Engagement }\end{array}$ & $\begin{array}{l}\text { Hauptmotivation: Lebensqualität der Bewohner verbessern } \\
(77 \% \text { / +4\%). } \\
\text { Positive Sekundärmotivation: Glauben in Wort und Tat le- } \\
\text { ben ( } 57 \% \text { / +13); Zugehörigkeit zur Kirche }(44 \% /+17 \%) \text {. } \\
\text { Negative Sekundärmotivation: neue Erfahrungen und Kom- } \\
\text { petenzen }(13 \% /-14 \%) \text {; Angelegenheiten selbst organisie- } \\
\text { ren }(2 \% \text { / }-13 \%) \text {. }\end{array}$ \\
\hline $\begin{array}{l}\text { Soziodemografische } \\
\text { Merkmale }\end{array}$ & $\begin{array}{l}\text { Überwiegend älter (70 Jahre und älter), weiblich, verwitwet, } \\
\text { evangelisch. }\end{array}$ \\
\hline $\begin{array}{l}\text { Kirchlicher Hinter- } \\
\text { grund }\end{array}$ & $\begin{array}{l}\text { Stark in Kirchengemeinde eingebunden, Engagement als } \\
\text { Teil kirchlichen Handelns. }\end{array}$ \\
\hline Tätigkeitsfelder & $\begin{array}{l}\text { Besuchsdienst, Spiritualität und Seelsorge, Gottesdienste } \\
\text { und Andachten. }\end{array}$ \\
\hline Dauer des Engagements & Sehr langes, teilweise jahrzehntelanges Engagement. \\
\hline Wege zum Ehrenamt & $\begin{array}{l}\text { Persönliche Ansprache durch Mitglieder der Kirchenge- } \\
\text { meinde. }\end{array}$ \\
\hline $\begin{array}{l}\text { Einschätzung der } \\
\text { Rahmenbedingungen } \\
\text { und besondere Heraus- } \\
\text { forderungen }\end{array}$ & Zufriedenheit mit den Rahmenbedingungen. \\
\hline $\begin{array}{l}\text { Möglichkeiten zur Ver- } \\
\text { besserung der Situation }\end{array}$ & $\begin{array}{l}\text { Mehr Einbindung, Anleitung und Begleitung, Stärkung der } \\
\text { Anerkennungskultur, Kooperation mit Kirchengemeinde, } \\
\text { Öffentlichkeitsarbeit. }\end{array}$ \\
\hline
\end{tabular}

Abb. 3: Beschreibung »Milieuverbunden Pflichtorientierte»

Bei der zweitgrößten Gruppe der Ehrenamtlichen in unserer Umfrage (mit einem Anteil von 30\%) kommen zu der klassischen Pflichtorientierung auch persönliche Motive hinzu. Ausgeprägt ist der Wunsch nach Eigeninitiative und eigenständiger Gestaltung des Engagements. Zusammenhänge zwischen Ehrenamt und Persönlichkeitsentwicklung lassen sich beobachten. Eine kirchliche Bindung spielt keine oder eher eine untergeordnete Rolle.

Eine dritte Gruppe von Ehrenamtlichen in der Evangelischen Heimstiftung (mit einem Anteil von 4\%) unterscheidet sich in einigen wichtigen Aspekten von den zuvor beschriebenen. Sie richten sich ausschließlich an eigenen Motiven aus. Sie wird darum als »rein selbstorientierte« Engagementgruppe betitelt.

Die vierte Gruppe der Ehrenamtlichen (mit einem Anteil von 8\%) zeigt einen fast aufopfernden Charakter. Von ihren Orientierungen und Einstellungen her ist sie nahe- zu ausschließlich an Pflichtwerten orientiert. So gut wie keine Bedeutung haben Zusammenhänge zwischen ehrenamtlicher Tätigkeit und der eigenen Person, haben Aspekte des sich selbst verwirklichen oder sich selbst weiterentwickeln. Der Begriff des »selbstlos Pflichtorientierten« trifft für diese Engagementgruppe zu.

Die Beschreibung der fünften Engagementgruppe (mit einem Anteil von 6\%) ist problematisch. Sie lässt sich nicht exakt einordnen. Keines der vorgeschlagenen Motive spricht die Befragten an. Haupt- und Sekundärmotivationen sind weit unterdurchschnittlich ausgeprägt. Offenbar treiben andersartige Gründe diese Engagementgruppe an; sie wird darum als »extern Motivierte« bezeichnet.

Schließlich bleibt eine letzte Engagementgruppe (mit einem Anteil von 9\%), deren Motive stark an eigenen Bedürfnissen und an Pflichtwerten orientiert sind. Sie kennzeichnet, dass wie in anderen Gruppen ausgeprägte Pflichtorientierung und persönliche Motive im Vordergrund stehen, Zusammenhänge zwischen Ehrenamt und Persönlichkeitsentwicklung sich aber nicht beobachten lassen. Eine kirchliche Bindung spielt keine Rolle. Sie wird als »selbst- und pflichtorientierte « Engagementgruppe bezeichnet (vgl. Abb. 4).

Die Darstellung der unterschiedlichen Formen und Typologien ehrenamtlichen Engagements zeigt ein komplexes Bild. Es lassen sich deutliche Differenzen zwischen den sechs Engagementgruppen mit zum Teil eindeutig voneinander abweichenden Orientierungen und Merkmalen feststellen. Auf organisatorischer Ebene wird es darauf ankommen, offen zu sein für unterschiedliche Formen und Motive, sie zu koordinieren und zu orchestrieren.

\section{Sechs Konsequenzen für das Freiwilligenmanagement}

Die ermittelten Ergebnisse liefern für das Freiwilligenmanagement wichtige Hinweise zur Gewinnung und zur Bindung von Ehrenamtlichen:

1. Gemeinsame Ziele in den Mittelpunkt stellen: Ei- nes der wichtigsten Ergebnisse der Analyse ist, dass Ehrenamtliche in der Evangelischen Heimstiftung sich an einem gemeinsamen Kern von Zielen orientieren: die Lebensqualität der Bewohner zu verbessern und dabei Spaß, Freude und Sinn erfahren zu können. Diese gemeinsame Zielsetzung, die Lebensqualität der Bewohner zu verbes- 


\begin{tabular}{|c|c|}
\hline Engagementgruppe & Selbst- und Pflichtorientierte \\
\hline Anteil & $9 \%$ \\
\hline $\begin{array}{l}\text { Motivbündel/Gründe } \\
\text { für ehrenamtliches } \\
\text { Engagement }\end{array}$ & $\begin{array}{l}\text { Hauptmotivation: Lebensqualität der Bewohner verbessern } \\
(94 \% \text { / +12\%). } \\
\text { Positive Sekundärmotivation: Tätigkeit, die Spaß und Freu- } \\
\text { de macht (74\% / + 19\%). } \\
\text { Negative Sekundärmotivation: neue Erfahrungen und Kom- } \\
\text { petenzen (12\% / -14\%); Angelegenheiten selbst organisie- } \\
\text { ren ( } 2 \% \text { / -13\%); Glauben in Wort und Tat leben (7\% / - } \\
37 \%) \text {; Zugehörigkeit zur Kirche ( } 2 \% /-25 \%) \text {. }\end{array}$ \\
\hline $\begin{array}{l}\text { Soziodemografische } \\
\text { Merkmale }\end{array}$ & $\begin{array}{l}\text { Häufiger in mittleren Jahren (50-59 Jahre), weiblich, selten } \\
\text { familiärer Bezug zum Heim, häufiger konfessionslos. (13\%). }\end{array}$ \\
\hline $\begin{array}{l}\text { Kirchlicher Hinter- } \\
\text { grund }\end{array}$ & $\begin{array}{l}\text { Kirchliche und religiöse Motive spielen keine oder eine sehr } \\
\text { untergeordnete Rolle. }\end{array}$ \\
\hline Tätigkeitsfelder & Persönliche Begleitung, Hauswirtschaft. \\
\hline Dauer des Engagements & Kürzer als in den Vergleichsgruppen. \\
\hline Wege zum Ehrenamt & Freunde und Bekannte, eigene Initiative. \\
\hline $\begin{array}{l}\text { Einschätzung der } \\
\text { Rahmenbedingungen } \\
\text { und besondere Heraus- } \\
\text { forderungen }\end{array}$ & Zufriedenheit mit der ehrenamtlichen Tätigkeit. \\
\hline $\begin{array}{l}\text { Möglichkeiten zur Ver- } \\
\text { besserung der Situation }\end{array}$ & $\begin{array}{l}\text { Mehr fachliche Unterstützung von den Hauptamtlichen, } \\
\text { Einführung in die Tätigkeit und Begleitung, Anerkennung }\end{array}$ \\
\hline
\end{tabular}

Abb. 4: Beschreibung Selbst- und Pflichtorientierte

Rahmenbedingungen laut, so klingen in der aktuellen Befragung Aspekte wie Kostenerstattung, Versicherungsschutz oder Anerkennung kaum an. Anzunehmen ist, dass die in der Diakonie geführte Diskussion um die Bedeutung des Ehrenamtes (2) dazu geführt hat, dass zentrale Aspekte aufgegriffen und geklärt wurden. Statt formaler Punkte stehen nun eher generelle Probleme im Vordergrund:

- Als ein zentraler Punkt muss die Frage nach Einführung und Begleitung der Ehrenamtlichen durch Hauptamtliche angesehen werden. Freiwillige erwarten, dass sie angeleitet, fachlich unterstützt, zum Teil kritisch begleitet werden. Sie erhoffen, dass Professionelle ihnen Sicherheit und Orientierung geben, Ansprechpartner und Vorbild sind, Wissen vermitteln, bei Problemen und Konflikten moderieren. Geklärt werden muss, wie die Zusammenarbeit mit Ehrenamtlichen in Stellenbeschreibung und Arbeitsplanung ihren Niederschlag finden. sern, lässt es gelingen, dass Ehrenamtliche aus sechs unterschiedlichen Engagementgruppen konkret vor Ort und mit hoher Zufriedenheit kooperieren. Im Zusammenhang aller Bemühungen, freiwillig Engagierte zu motivieren und zu gewinnen, ist es wichtig, diese Besonderheit hervorzuheben.

2 Ressourcen bündeln und Netzwerke gestalten: Über • 60 Prozent der Ehrenamtlichen sind neben ihrem Engagement in der Evangelischen Heimstiftung auch noch in anderen Bereichen aktiv und bringen so viele Kontakte mit ins Heim. Durch Nutzung dieser Potenziale ergeben sich erweiterte Chancen der Öffnung der Einrichtung in das Gemeinwesen. Zentrale Aufgabe der Einrichtungen ist es, alle vorhandenen Ressourcen zu erschließen und zu bündeln.

3. Engagementbereite Personen gezielt ansprechen: Wer Ehrenamtliche gewinnen möchte, muss wissen, wie diejenigen, die bereits aktiv sind, ihren Weg zum Engagement gefunden haben. Überwiegend erfolgt der Zugang zum Ehrenamt in der Evangelischen Heimstiftung über persönliche Bezüge. Gelegenheiten ergeben sich durch bereits bestehende Kontakte, sei es mit Angehörigen und Freunden, durch berufliche Verbindungen, im Kontext der Kirchengemeinde oder Kommune.

4. Engagement fördernde Rahmenbedingungen schaffen: Wurde bei der letzten Ehrenamtsbefragung in der Evangelischen Heimstiftung vor sieben Jahren auf die Frage, was denn die Situation der Ehrenamtlichen verbessern könnte, vor allem Forderungen nach verbesserten
- Ein weiterer zentraler Punkt ist die Frage der Mitsprachemöglichkeiten. Je jünger Ehrenamtliche sind, desto entscheidender wird das Partizipationsmoment. Partizipation bedeutet, eigene Schwerpunkte in der ehrenamtlichen Arbeit festlegen, Neues in Angriff nehmen zu können. Hierin steckt ein besonderer Anreiz für Engagementgruppen, für die Eigeninitiative und Selbstorganisation besonders wichtig erscheinen. Möglichkeiten zur Partizipation wie auch der Raum zur Realisierung eigener Ideen erfahren viele Freiwillige als individuelle Anerkennung und Wertschätzung.

- Ein dritter zentraler Punkt betrifft die Aspekte Anerkennung sowie Fort- und Weiterbildung. Beide Aspekte sind wichtig, wenn es darum geht, Engagierte zu gewinnen und zu binden. Die Ergebnisse zeigen kritisch, dass die über 70-Jährigen deutlich weniger mit Formen der Anerkennung bedacht werden als die jüngeren Altersgruppen. Auch muss die Form der Anerkennung zur Person passen. Darüber hinaus vermissen jüngere Ehrenamtliche adäquate Fortbildungsangebote. Wer Wert darauf legt, neue und jüngere Leute zu gewinnen, wird darauf achten, dass attraktive Angebote gemacht und genutzt werden.

5 Rollen von Haupt- und Ehrenamtlichen klären: Bei - nahezu allen Engagementgruppen hat sich die Frage als bedeutsam herausgestellt, welche Rolle Ehrenamtliche haben und welche Funktion den Hauptamtlichen dabei zukommt. Vonseiten der freiwillig Mitarbeitenden werden diese Fragen gestellt - und auch für die beruflich Mitarbeitenden muss es in deren Interesse liegen, die je- 
weiligen Aufgaben und Rollen zu definieren. Mit Hauptund Ehrenamtlichen treffen verschiedene Sichtweisen, widerstreitende Wertvorstellungen und vielfältiges fachliches Know-how aufeinander. Will man eine Bündnispartnerschaft zwischen Profis und Freiwilligen installieren, geht dies über die im Grundmodell (vgl. Kasten »Was man unter >Freiwilligenmanagement $<$ heute verstehen kann«) skizzierten Maßnahmen, Prozesse und Instrumente weit hinaus. Es geht um mehr als nur Freiwillige zu gewinnen, sie zu binden und wieder zu verabschieden. Es braucht dazu eine spezifische Kultur der Organisation, ein reflektiert professionelles Selbstverständnis, die Bereitschaft, sich als Institution in den Sozialraum zu öffnen, Einmischung zu wünschen und Menschen etwas zuzutrauen. Voraussetzung dafür ist, dass vonseiten des Managements Soziale Arbeit als Koproduktion gewollt wird, Beteiligung und Partizipation erwünscht sind, Räume und Zeit für eine Dialog- und Streitkultur eingeplant sind.

6. Werbung und Öffentlichkeitsarbeit intensivieren: Neben der gezielten persönlichen Ansprache ist eine Ausweitung vorhandener Freiwilligenpotenziale insbesondere durch verstärkte Öffentlichkeitsarbeit möglich. Dies ist die Meinung vieler Befragter. Werbung für ehrenamtliches Engagement sollte zum einen den Nutzen herausstellen, den pflegebedürftige Menschen, ihre Bezugspersonen und die Gesellschaft haben. Zum anderen sollte sie betonen, dass auch die Einzelnen erheblich von ihrem Engagement profitieren, etwa durch Begleitung und Supervision oder in Form attraktiver Fortbildungsmöglichkeiten. Ob Menschen sich tatsächlich in der stationären Pflege für andere engagieren, hängt aber auch davon ab, ob ihr Engagement gesellschaftlich akzeptiert wird. Solange negativ akzentuierte Altersbilder dominieren, Skandalisierung und Schuldzuweisung das mediale Bild der stationären Altenhilfe beherrschen, werden viele auf ein Engagement in diesem Bereich verzichten. Werbung und Öffentlichkeitsarbeit greifen aber nicht nur Bilder auf, sie können Altersbilder auch modifizieren oder neue schaffen. Dargestellt werden kann, wie Ehrenamtliche zur Erhöhung der Lebensqualität pflegebedürftiger Menschen beitragen und Perspektiven in Situationen vermitteln, denen viele Menschen mit großen Ängsten entgegensehen. Wünschenswert wäre, ehrenamtlich Engagierten die Teilhabe an der Gestaltung medialer Botschaften zu ermöglichen, damit sie selbst einen Beitrag für ein realistisches Altersbild leisten.

\section{Anmerkungen}

(1) Die umfangreichste Studie ist der im Auftrag des Bundesministeriums für Familien, Senioren, Frauen und Jugend erstellte Freiwilligensurvey (mit rund 15.000 Befragten), der erstmals 1999 erstellt und im Jahr 2004 wiederholt wurde (T. Gensicke 2004). Für Baden-Württemberg liegt eine Landesauswertung vor (H. Hoch u. a. 2006). Eine wichtige Vergleichsstudie ist eine Befragung bürgerschaftlich Engagierter in der stationären Altenhilfe in Baden-Württemberg (T. Klie u. a. 2005).

(2) »Handeln Ehrensache« lautete der Slogan einer Kampagne, in der sich die Diakonie in den Jahren 2001 und 2002 intensiv mit der Frage des Ehrenamtes auseinandergesetzt hat.

\section{Ausblick}

Freiwilliges Engagement braucht eine förderliche Infrastruktur. Die Potenziale engagementbereiter Menschen sind bei weitem noch nicht ausgeschöpft. Systematisch und langfristig angelegte Maßnahmen des Freiwilligenmanagements werden vermehrt Beachtung finden. In der Bündnispartnerschaft von Haupt- und Ehren-amtlichen liegt eine - für eine Gesellschaft des langen Lebens höchst bedeutsame Ressource. Passgenaue Angebote sind gefragt, die ausdrücklich Interessen und Motive von Ehrenamtlichen ansprechen. Wesentlich ist eine Anerkennungskultur, die zur Person passt.

Vieles deutet darauf hin, dass Freiwillige künftig noch stärker ein integraler Bestandteil des sozialen Systems sein werden. Systematisch angelegte Maßnahmen des Freiwilligenmanagements werden damit vermehrt Beachtung finden.

\section{Literatur}

BMFSFJ (Bundesministerium für Familie, Senioren, Frauen und Jugend) (Hg.), Fünfter Bericht zur Lage der älteren Generation in der Bundesrepublik Deutschland. Potenziale des Alters in Wirtschaft und Gesellschaft. Der Beitrag älterer Menschen zum Zusammenhalt der Generationen, Berlin 2005.

Biedermann, Christiane, Was heißt Freiwillige managen? Grundzüge des Freiwilligen-Managements, in: Nährlich, Stefan/Zimmer, Annette (Hg.), Management in Nonprofit-Organisationen. Eine praxisorientierte Einführung, Opladen 2000, 107-128.

Gensicke, Thomas, Freiwilliges Engagement in Deutschland 1999-2004. Ergebnisse der repräsentativen Trenderhebung zu Ehrenamt, Freiwilligenarbeit und bürgerschaftlichem Engagement. Vorgelegt von TNS Infratest Sozialforschung, München 2004.

Hoch, Hans/Klie, Thomas/Wegner, Martina, Bürgerschaftliches Engagement in Baden-Württemberg. Landesauswertung zu den Ergebnissen des 2. Freiwilligensurvey 2004, Freiburg im Breisgau 2006.

Kaltenbrunner, Katharina Anna, Freiwilligenmanagement in (großen) Sport- \& Gesundheitsvereinen. Gesellschaftliche Wandlungsprozesse und deren Implikationen für die Gestaltung der Freiwilligenarbeit, in: Urnik, Sabine (Hg.), Sport und Gesundheit in Wirtschaft und Gesellschaft, Wien 2007, 79-99.

Klie, Thomas/Hoch, Hans/Pfundstein, Thomas, BELA Bürgerschaftliches Engagement für Lebensqualität im Alter. Schlussbericht zur »Heim- und Engagiertenbefragung «, Freiburg im Breisgau 2005.

Mayerhofer, Helene, Der Stellenwert Ehrenamtlicher als Personal in Nonprofit Organisationen, in: Eckardstein, Dudo v./Ridder, Hans-Gerd (Hg.), Personalmanagement als Gestaltungsaufgabe im Nonprofit und Public Management, München 2003, 97-118.

McCurley, Steve/Lynch, Rick, Essential Volunteer Management. 2. Auflage. London 1998. 


\section{Was man unter "Freiwilligenmanagement« heute verstehen kann}

Versteht man unter ehrenamtlichem, freiwilligem, bürgerschaftlichen Engagement ein frei gewähltes, unentgeltliches Tätigsein im Rahmen einer Organisation zugunsten anderer und der Gemeinschaft, und versteht man Ehrenamtliche grundsätzlich als freiwillig mitarbeitendes »Personal« einer Einrichtung $(\mathrm{H}$. Mayerhofer 2003, 114), lassen sich für die Freiwilligenarbeit »entsprechende Praktiken des Personalmanagements « (116) - wenn auch in adaptierter Form anwenden. der Diakonie: stark wertorientierten) Zielsetzungen der Organisation.

In Anlehnung an das aus den USA stammende Urbild eines »Volunteer Managements « von S. Mc.Curley und R. Lynch $(1998,19)$ und an prozessorientierte Freiwilligenmanagement-Konzeptionen von C. Biedermann (in: T. Kegel 2002 , 95) und K. A. Kaltenbrunner (2007, 95), ergibt sich ein Grundmodell für eine Freiwilligenmanagement-Konzeption (vgl. Abb.).

\section{Grundmodell zum Freiwilligenmanagement}
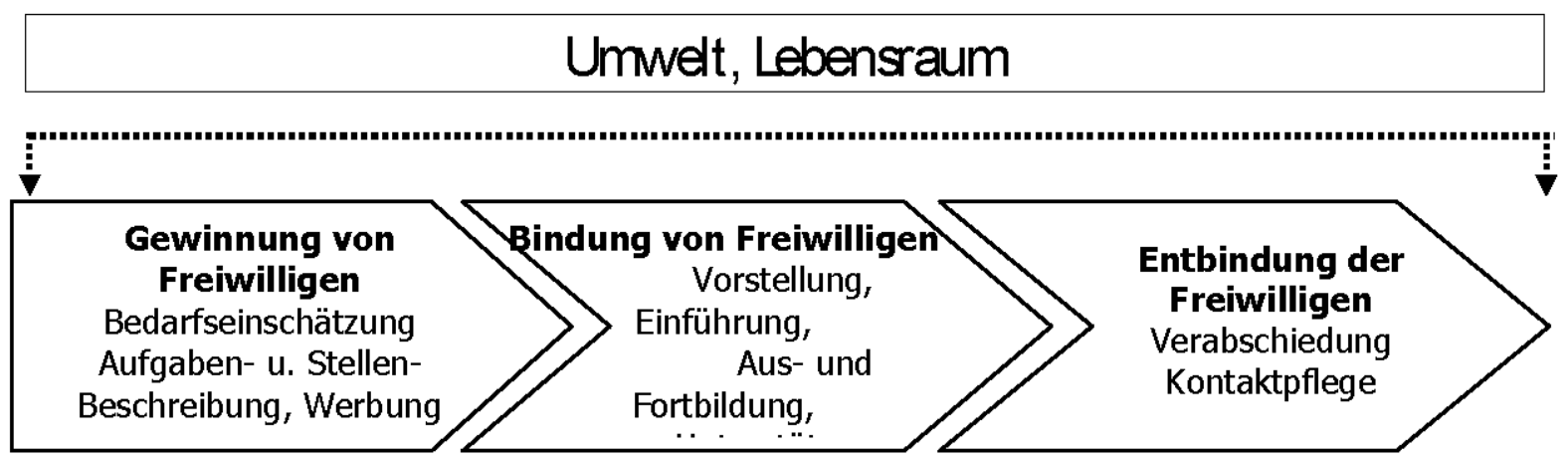

interne Strukturen, Prozesse, Interaktionen

Enbeziehung der hauptamtlichen Mitarbeitenden

Vernetzung mit übergeorcheten Management systemen

Analog von Aufgaben des Personalmanagements ist unter Freiwilligenmanagement die systematische Gestaltung, Steuerung und Entwicklung der Arbeit mit Freiwilligen zu verstehen, die eine erfolgreiche Führung von Freiwilligen einschließt. Ziel des Freiwilligenmanagements muss sein, ehrenamtliches Engagement als bedarfsgerechte Dienstleistung hier für pflegebedürftige Menschen - zu verbinden mit Erwartungen und unterschiedlichen Motivationsstrukturen der Freiwilligen als auch mit den (im Bereich
Das Grundmodell gliedert sich in drei Kernprozesse der Gewinnung freiwilliger Mitarbeiter, der Freiwilligenbindung und Freiwilligenentbindung. In jeder einzelnen Phase spielen Bedürfnisse, Erwartungen und Interessen der freiwillig Engagierten eine zentrale Rolle. Detaillierte einzelne Schritte des Freiwilligenmanagements sowie Aufgabenbereichen eines »Freiwilligenkoordinators « sind ausführlich bei C. Biedermann (2000, 110-126; 2002, 81-85) dargestellt.

Thomas Mäule 